\title{
Numerical Comparisons for the Null Distribution of the Bagai Statistic
}

\author{
Hyung-Tae $\mathrm{Ha}^{1, a}$ \\ ${ }^{a}$ Department of Applied Statistics, Gachon University
}

\begin{abstract}
Bagai et al. (1989) proposed a distribution-free test for stochastic ordering in the competing risk model, and recently Murakami (2009) utilized a standard saddlepoint approximation to provide tail probabilities for the Bagai statistic under finite sample sizes. In the present paper, we consider the Gaussian-polynomial approximation proposed in Ha and Provost (2007) and compare it to the saddlepoint approximation in terms of approximating the percentiles of the Bagai statistic. We make numerical comparisons of these approximations for moderate sample sizes as was done in Murakami (2009). From the numerical results, it was observed that the Gaussianpolynomial approximation provides comparable or greater accuracy in the tail probabilities than the saddlepoint approximation. Unlike saddlepoint approximation, the Gaussian-polynomial approximation provides a simple explicit representation of the approximated density function. We also discuss the details of computations.
\end{abstract}

Keywords: The Bagai statistic, null distribution, numerical comparisons, Gaussian-polynomial approximation, saddlepoint approximation, cumulant generating function, moments.

\section{Introduction}

The important procedure in connection with nonparametric test statistics is to determine their null distributions, from which percentiles can be obtained. Even though combinatorial methods are widely utilized in order to calculate probabilistic quantities for distribution-free nonparametric test statistics, it should be pointed out that the combinatorial methods which involve all the possible combinations of the outcomes, are very complicated and time consuming as the sample size increases. Accordingly approximation methods are necessary to allow easy computational implementation, and asymptotic and approximation techniques should play essential roles in nonparametric testing. Recently, various higher order asymptotic approximation methods for nonparametric statistics such as Edgeworth expansion, normal, saddlepoint and uniform approximations have been discussed for numerical comparisons in terms of computational accuracy and efficiency, see for instance Bean et al. (2004).

In this paper, we consider three approximations to the null-distribution of the Bagai test statistic, which is a distribution-free test for stochastic ordering in the competing risk model. The three approximation methods are the Bagai approximation, the saddlepoint approximation and the Gaussianpolynomial approximation. Bagai et al. (1989) provided numerical comparisons with the normal approximation and the exact distribution, and Mukarami (2009) utilized the saddlepoint approximation which is based on the cumulant generating function of the Bagai statistic and compared it to the Bagai approximation. For fair numerical comparisons of those three approximation methods, the

This research was supported by Basic Science Research Program through the National Research Foundation of Korea(NRF) funded by the Ministry of Education, Science and Technology (2011-0013791).

${ }^{1}$ Department of Applied Statistics, Gachon University, Seongnam, Kyonggi-Do 461-701, Korea.

E-mail: htha@gachon.ac.kr 
cases involving moderate sample sizes for $5 \leq n \leq 20$, which was used in Murakami (2009), are revisited.

The saddlepoint approximations proposed by Daniels $(1954,1987)$ and developed by Lugannani and Rice (1980) can be applied to any statistic that admits a cumulant generating function. The saddlepoint method is well known to generate accurate tail probabilities, even for small sample sizes. Saddlepoint approximations have been used with great success by many authors, and excellent discussions of their applications to a range of distributional problems can be found for instance Easton and Ronchetti (1986), Reid (1988), Jensen (1995), Goutis and Casella (1999), Huzurbazar (1999), Kolassa (2006) and Butler (2007). But it should be mentioned that the saddlepoint approximation is appropriate for the tail areas of the null distributions. It should also be pointed out that the saddlepoint method requires a numerical method such as the Newton-Raphson algorithm to calculate the saddlepoint, which is obtained by inverting the derivative of the cumulant generating function of interest. For this reason, saddlepoint method could be crude when the cumulant generating functions are complicated.

In this paper, we present a simple moment-based approximation method, namely the Gaussianpolynomial approximation, which was originally proposed by Ha and Provost (2007). It will be shown that this approach can be successfully utilized to provide accurate percentiles of the Bagai statistic and that is also very flexible and quickly adapt to the features of the target distributions. In addition, we are interested in numerical comparisons with respect to the performance and efficiency of other approximation methods. As explained in Ha and Provost (2007), the Gaussian-polynomial approximation is based on a moment-matching technique. Since the higher order moments can easily be obtained numerically by differentiating the moment generating function, the Gaussian-polynomial approximation can be readily utilized. It should be pointed out that the Gaussian-polynomial approximation can be considered as a refinement of the Bagai approximation which adapts missing features of the target distribution by utilizing more than the first two moments that are used in the Bagai approximation.

In Section 2, the Bagai statistic is briefly introduced including its moment generating function and its moments. In Section 3, a brief introduction to the main approximation methods including the Bagai, saddlepoint and Gaussian-polynomial approximations, is provided. In Section 4, we describe in detail steps required to construct an optimal Gaussian-polynomial approximant and provide numerical comparisons for the approximations to the null distribution of the Bagai statistic for various combinations of two independent sample sizes. As well, relevant computational issues are discussed.

\section{The Bagai Statistic}

In this section, the Bagai statistic is briefly introduced. This statistic considers the competing risks set-up wherein a unit is subject to failure due to one of two risks. We denote by $X$ and $Y$ the notional lifetimes of a unit under these two risks and $X_{1}, \ldots, X_{n}$ and $Y_{1}, \ldots, Y_{n}$ individuals in the two random samples of independent observations of size $n$ from two continuous distributions, $F(\cdot)$ and $G(\cdot), T=$ $\operatorname{Min}(X, Y)$ is the time at which the unit fails and $T_{i}=\operatorname{Min}\left(X_{i}, Y_{i}\right)$ is the observed time to failure for the $i^{\text {th }}$ sample. On letting $\delta=\mathcal{I}(X>Y)$ be the indicator for the cause of failure, we can observe $\left(T_{1}, \delta_{1}\right), \ldots,\left(T_{n}, \delta_{n}\right)$ where $\delta_{i}=\mathcal{I}\left(X_{i}>Y_{i}\right)$ is an indicator for the $i^{t h}$ unit. On the basis of these data types, we are interested in testing the following hypothesis:

$$
H_{0}: F(x)=G(x) \text { against } \quad H_{A}: F(x) \leq G(x) .
$$


Further denoting $R_{i}$ the rank of $T_{i}$ among $T_{1}, \ldots, T_{n}$, Bagai et al. (1989) proposed a test statistic

$$
\mathcal{S}=2 \sum_{i=1}^{n}\left(2 n-1-R_{i}\right) \delta_{i}-\frac{3 n(n-1)}{2} .
$$

Bagai et al. (1989) provided the explicit expressions for the important statistical quantities such as the mean and variance

$$
E(\mathcal{S})=0 \quad \text { and } \quad \operatorname{Var}(\mathcal{S})=\frac{n(n-1)(14 n-13)}{6}
$$

under the null hypothesis as well as the moment generating function $\mathcal{M}(t)$ corrected by Murakami (2009) as follows:

$$
\mathcal{M}(t)=2^{-n} \exp \left(\frac{3 n(n-1) t}{2}\right) \prod_{j=2}^{n+1}[1+\exp (2 t(2 n-j))]
$$

\section{Approximations}

Three approximation methods are briefly reviewed in this section. First, the Bagai approximation is simply a normal approximation on the basis of the first two moments of the distribution. The saddlepoint approximation makes use of the cumulant generating function. When higher order moments can easily be computed numerically by differentiating the moment generating function, such moments can be utilized in moment based approximation methods such as the Gaussian-polynomial approximation. The Gaussian-polynomial approximation can be considered as an extension of the Bagai approximation which utilizes more moments in order to refine the Bagai approximation by making use of a polynomial adjustment.

\subsection{The Bagai approximation}

Given the expected value and variance of the Bagai statistic given in Equation (2.3), the Bagai approximation, denoted by $f_{B}(x)$, can simply be expressed as the following normal density function:

$$
f_{B}(x)=\frac{1}{\sqrt{2 \pi \operatorname{Var}(\mathcal{S})}} \exp \left(-\frac{(x-E(\mathcal{S}))^{2}}{2 \operatorname{Var}(\mathcal{S})}\right) .
$$

\subsection{The Saddlepoint approximation}

We briefly describe the saddlepoint approximation proposed by Daniels $(1954,1987)$ and the formula developed by Lugannani and Rice (1980), from which accurate approximated tail percentiles to the distribution of the Bagai statistic can be obtained. The cumulant generating function of the $\mathcal{S}$ statistic denoted by $\kappa(s)$ can be easily obtained by taking logarithm on the moment generating function $\mathcal{M}(s)$ of the Bagai statistic $\mathcal{S}$, that is,

$$
\kappa(s)=\log [\mathcal{M}(s)]=-n \log 2-\frac{3 n(n-1) s}{2}+\sum_{j=2}^{n+1} \log [1+\exp (2 s(2 n-j))] .
$$


The first two derivatives of its cumulant generating function are as follows:

$$
\begin{aligned}
\kappa^{(1)}(s) & =-\frac{3 n(n-1)}{2}+\sum_{j=2}^{n+1} \frac{2(2 n-j) \exp (2 s(2 n-j))}{1+\exp (2 s(2 n-j)}, \\
\kappa^{(2)}(s) & =\sum_{j=2}^{n+1} \frac{4(j-2 n)^{2} \exp (2 s(j+2 n))}{[\exp (2 j s)+\exp (4 n s-j)]^{2}},
\end{aligned}
$$

where $\kappa^{(i)}(\cdot)$ denotes the $i^{\text {th }}$ derivative. When a unique solution $(s=\hat{s})$ is obtained from equating $\kappa^{(1)}(s)=v$, the saddlepoint approximation to the probability density function denoted by $f_{S P}(x)$ is

$$
f_{S P}(x)=\sqrt{2 \pi \kappa(\hat{s})} \exp (\kappa(\hat{s})-x \hat{s}) .
$$

Since the saddlepoint approximation to the probability density function is not an integrable function, the generalized Lugannani and Rice formula for nonnormal distributions suggested by Wood $e t$ al. (1993) to determine the saddlepoint approximation to $\operatorname{Pr}(\mathcal{S} \geq v)$ is

$$
\operatorname{Pr}(\mathcal{S} \geq v) \approx 1-\Phi(\hat{w})+\phi(\hat{w})\left(\frac{1}{\hat{u}}-\frac{1}{\hat{w}}\right),
$$

where $\hat{w}=\sqrt{2(\hat{s} v-\kappa(\hat{s}))} \operatorname{sgn}(\hat{s}), \hat{u}=\hat{s} \sqrt{\kappa^{(2)}(\hat{s})}, \operatorname{sgn}(\hat{s})= \pm 1,0$ if $\hat{s}$ is positive, negative, or zero, and $\phi(\cdot)$ is the standard normal density function and $\Phi(\cdot)$ is the corresponding cumulative distribution function.

\subsection{The Gaussian-polynomial approximation}

We utilize a general semi parametric approach, the so-called Gaussian-polynomial approximation, which was proposed in Ha and Provost (2007). Let $S$ be a random variable whose raw moments $E\left(S^{h}\right)$ be denoted by $\mu_{S}(h), h=0,1, \ldots$. We are interested in approximating the probability density functions of the random variable $S$. A polynomially adjusted Gaussian approximation of degree $d$, denoted by $f_{d}(x)$, is

$$
f_{d}(x)=g(x) \sum_{i=0}^{d} \theta_{i} x^{i}
$$

This approximant is expressed as the product of the Bagai approximation and a polynomial adjustment. The Gaussian approximation obtained on the basis of the first two moments is

$$
g(x)=\frac{1}{\sqrt{2 \pi \sigma_{g}^{2}}} \exp \left(-\frac{\left(x-\mu_{g}\right)^{2}}{2 \sigma_{g}^{2}}\right),
$$

where the parameters $\mu_{g}$ and $\sigma_{g}^{2}$ are determined from the first two moments of the target distribution as $\mu_{g}=\mu_{S}(1)$ and $\sigma_{g}^{2}=\mu_{S}(2)-\left(\mu_{S}(1)\right)^{2}$.

By matching the first $d$ moments obtained from Equation (3.7) to those of $\mathcal{S}$, we can obtain the coefficients $\theta_{i}$ of the polynomial adjustment. Accordingly, the coefficients $\theta_{i}$ are such that the 
following matrix form equation:

$$
\left(\begin{array}{c}
\theta_{0} \\
\theta_{1} \\
\vdots \\
\theta_{d}
\end{array}\right)=\left(\begin{array}{ccccc}
\mu_{g}(0) & \mu_{g}(1) & \cdots & \mu_{g}(d-1) & \mu_{g}(d) \\
\mu_{g}(1) & \mu_{g}(2) & \cdots & \mu_{g}(d) & \mu_{g}(d+1) \\
\vdots & \vdots & \ddots & \vdots & \vdots \\
\mu_{g}(d) & \mu_{g}(d+1) & \cdots & \mu_{g}(2 d-1) & \mu_{g}(2 d)
\end{array}\right)^{-1}\left(\begin{array}{c}
1 \\
\mu_{S}(1) \\
\vdots \\
\mu_{S}(d)
\end{array}\right),
$$

where $\mu_{g}(h)$ is the $h^{\text {th }}$ moment of the corresponding Gaussian approximation obtained on the basis of matching the first two moments. It should be noted that since the Gaussian-polynomial density approximant has a simple explicit representation, the corresponding distribution approximant, denoted by $F_{d}(w)$, can easily be obtained by numerical integration. The continuity correction should be considered to approximate nonparametric discrete distributions via continuous function. Since the Bagai statistic takes on successive integer values, the Gaussian-polynomial distribution approximant is taken to be

$$
F_{d}(w)=\int_{-\infty}^{w+\frac{1}{2}} f_{d}(x) \mathrm{d} x .
$$

When using this approach, an important step is the determination of optimal degree of the polynomial adjustment. Several methods have been proposed to determine the optimal degree, see for instance Ha and Provost (2007) and Provost et al. (2009). A suitable degree for a density approximation can be determined by a de visu inspection of the density plots of approximants of successive degrees, as explained in Ha and Provost (2007). More specifically, one would be satisfied that a density approximant of degree $d$ is adequate if no noticeable differences between approximants of successive orders are observed. Provost et al. (2009) proposed making use of measures of discrepancy such as the integrated squared differences between density or distribution approximants of successive degrees. Entropy minimization could also be considered as an alternative for optimal degree selection. Since an appropriate optimal degree selection criterion should be chosen according to the purpose of the use of the approximation methods, a de visu approach would be appropriate. Since our purpose is numerical comparisons between the saddlepoint and Gaussian-polynomial approximations, we are aiming via a de visu rule to show that the Gaussian-polynomial approximation can outperform or at least be comparable to saddlepoint approximation in terms of approximation accuracy in the null distribution of the Bagai statistic. That is, we stop increasing the degree of polynomial adjustment of the Gaussian-polynomial approximation when it seems reasonable to posit that it outperforms the sadddepoint approximation. It should be noted that the null odd moments of the symmetric target distribution do not contribute to increasing in any way the precision of the Gaussian-polynomial approximation. This can be inferred from the fact that the odd degree coefficients of a polynomial adjustment are zero. More details in connection with approximating the symmetric distribution are described in $\mathrm{Ha}$ (2007). Therefore, in connection with the use of a de visu rule, the accuracy of saddlepoint approximation shall be taken to be the tolerance for the Gaussian-polynomial approximation and two degrees shall be added to the polynomial adjustment when the approximant used is deemed to not provide enough precision. This rule was implemented in the numerical examples considered in the next section.

\section{Numerical Comparisons}

\subsection{Gaussian-polynomial approximation}

In this subsection, we describe in detail the steps needed to construct a Gaussian-polynomial approximation. Consider the Bagai statistic when $n=8$. The first six exact moments of the Bagai statistic 
Table 1: Numerical results for the $1 \%$ significance level

\begin{tabular}{cccccc}
\hline \hline$n$ & $\mathrm{~S}$ & Exact & Bagai & Saddlepoint & GP(4) \\
\hline 7 & 51 & 0.0156 & 0.0183 & 0.016256 & 0.016465 \\
8 & 68 & 0.0117 & 0.0126 & 0.010587 & 0.010849 \\
9 & 84 & 0.0117 & 0.0113 & 0.009430 & 0.009659 \\
10 & 99 & 0.0107 & 0.0117 & 0.010029 & 0.010216 \\
11 & 115 & 0.0102 & 0.0118 & 0.010393 & 0.010548 \\
12 & 134 & 0.0105 & 0.0186 & 0.009538 & 0.009672 \\
13 & 152 & 0.0102 & 0.0109 & 0.009701 & 0.009814 \\
14 & 169 & 0.0106 & 0.0116 & 0.010537 & 0.010634 \\
15 & 191 & 0.0103 & 0.0107 & 0.009674 & 0.009759 \\
16 & 210 & 0.0105 & 0.0111 & 0.010161 & 0.010235 \\
17 & 232 & 0.0100 & 0.0181 & 0.009895 & 0.009961 \\
18 & 255 & 0.0100 & 0.0105 & 0.009595 & 0.009654 \\
19 & 275 & 0.0104 & 0.0110 & 0.010205 & 0.010257 \\
20 & 298 & 0.0104 & 0.0109 & 0.010201 & 0.010248 \\
\hline \hline
\end{tabular}

when $n=8$, that is, $\mu_{\mathcal{S}}(h), h=1,2, \ldots, 6$, which can be numerically obtained by differentiating its moment generating function, are 0,924, 0, 2310504, 0 and 8661316704. Note that all the odd moments are zero since the Bagai statistic is symmetric about the point of zero. The two parameters $\mu_{g}$ and $\sigma_{g}^{2}$ of normal distribution given in Equation (3.8) are determined from the first two moments $\mu_{\mathcal{S}}(1)=0$ and $\mu_{\mathcal{S}}(2)=924$, that is, $\mu_{g}=0$ and $\sigma_{g}^{2}=924$. It should be noted that this normal distribution is in fact the Bagai approximation. Since the Bagai approximation can not be considered to outperform saddlepoint approximation as can be seen in Table 2, we utilize the polynomially adjusted Gaussian approximation by increasing the power of polynomial adjustment by two degrees at once. It is observed that the $4^{\text {th }}$ degree Gaussian-polynomial approximation can be considered to outperform saddlepoint approximation, as can be seen in Table 2, because out of the 14 cases corresponding to $7 \leq n \leq 20$, which were presented in Murakami (2009), the $4^{\text {th }}$ degree Gaussian-polynomial approximation provides more accuracy than the saddlepoint approximation in 12 cases except for $n=7$ and $n=11$. (Note that the $4^{\text {th }}$ degree Gaussian-polynomial approximation outperforms the saddlepoint approximation when $n=9$ and $n=15$ ). As determined from Equation (3.8), we could obtain the following fourth-degree Gaussian-polynomial density approximant to the density function of the Bagai statistic:

$$
\begin{aligned}
f_{4}(x) & =g(x) \sum_{i=0}^{4} \theta_{i} x^{i} \\
& =0.0131242 \exp \left[-0.000541126 x^{2}\right]\left(0.963277+0.0000794865 x^{2}-1.43374 \times 10^{-8} x^{4}\right) .
\end{aligned}
$$

Dashed line in Figure 2 shows the source of improvement of the fourth-degree Gaussian-polynomial density approximant.

\subsection{Approximating the $1 \%$ significance level}

Table 1 shows the numerical results for the saddlepoint and fourth degree Gaussian-polynomial approximations in the case of a $1 \%$ significance level in addition to the exact probability and Bagai approximation calculated in Bagai et al. (1989). The differences between the exact probability of the Bagai statistic and the approximations are given in Table 2. In Tables $1 \sim 4$, we denote by $\mathcal{S}$ and $n$ the exact critical value of the Bagai statistic and the sample size, respectively. The bolded numbers represent the minimum difference between the exact and approximate values. We make comparisons for 
Table 2: Difference between the exact values and approximations

\begin{tabular}{ccccc}
\hline \hline$n$ & Bagai & Saddlepoint & GP(2) & GP(4) \\
\hline 7 & 0.0027 & $\mathbf{0 . 0 0 0 6 5 6}$ & 0.002673 & 0.000865 \\
8 & 0.0009 & 0.001113 & 0.000942 & $\mathbf{0 . 0 0 0 8 5 1}$ \\
9 & $\mathbf{0 . 0 0 0 4}$ & 0.002270 & 0.000429 & 0.002041 \\
10 & 0.0010 & 0.000671 & 0.000958 & $\mathbf{0 . 0 0 0 4 8 4}$ \\
11 & 0.0016 & $\mathbf{0 . 0 0 0 1 9 3}$ & 0.001653 & 0.000348 \\
12 & 0.0081 & 0.000962 & $\mathbf{0 . 0 0 0 3 7 5}$ & 0.000828 \\
13 & 0.0007 & 0.000499 & 0.000723 & $\mathbf{0 . 0 0 0 3 8 6}$ \\
14 & 0.0010 & 0.000063 & 0.001055 & $\mathbf{0 . 0 0 0 0 3 4}$ \\
15 & $\mathbf{0 . 0 0 0 4}$ & 0.000626 & 0.000418 & 0.000541 \\
16 & 0.0006 & 0.000339 & 0.000632 & $\mathbf{0 . 0 0 0 2 6 5}$ \\
17 & 0.0081 & 0.000105 & 0.008919 & $\mathbf{0 . 0 0 0 0 3 9}$ \\
18 & 0.0005 & 0.000405 & 0.000452 & $\mathbf{0 . 0 0 0 3 4 6}$ \\
19 & 0.0006 & 0.000195 & 0.000611 & $\mathbf{0 . 0 0 0 1 4 3}$ \\
20 & 0.0005 & 0.000199 & 0.000564 & $\mathbf{0 . 0 0 0 1 5 2}$ \\
\hline \hline
\end{tabular}

Table 3: Numerical results for the $5 \%$ significance level

\begin{tabular}{cccccc}
\hline \hline$n$ & S & Exact & Bagai & Saddlepoint & GP(10) \\
\hline 5 & 22 & 0.0625 & 0.0522 & 0.057870 & 0.057443 \\
6 & 31 & 0.0625 & 0.0499 & 0.051279 & 0.05114 \\
7 & 41 & 0.0547 & 0.0463 & 0.047075 & 0.047001 \\
8 & 50 & 0.0508 & 0.0499 & 0.050831 & 0.050825 \\
9 & 62 & 0.0508 & 0.0461 & 0.046546 & 0.046541 \\
10 & 73 & 0.0508 & 0.0472 & 0.047642 & 0.047648 \\
11 & 83 & 0.0527 & 0.0513 & 0.051902 & 0.051920 \\
12 & 98 & 0.0500 & 0.0467 & 0.046961 & 0.046970 \\
13 & 108 & 0.0528 & 0.0516 & 0.052143 & 0.052158 \\
14 & 123 & 0.0511 & 0.0494 & 0.049753 & 0.049765 \\
15 & 137 & 0.0516 & 0.0495 & 0.049831 & 0.049841 \\
16 & 150 & 0.0523 & 0.0512 & 0.051650 & 0.051661 \\
17 & 166 & 0.0519 & 0.0500 & 0.050448 & 0.050456 \\
18 & 181 & 0.0516 & 0.0506 & 0.050878 & 0.050886 \\
19 & 197 & 0.0516 & 0.0505 & 0.050748 & 0.050755 \\
20 & 214 & 0.0511 & 0.0499 & 0.050178 & 0.050185 \\
\hline \hline
\end{tabular}

the tail probabilities of the Bagai statistic between the saddlepoint and Gaussian-polynomial approximations. The fourth degree Gaussian-polynomial approximant provides the minimum differences in 9 cases among those four approximants and outperforms saddlepoint approximation in 12 cases out of 14 cases. It shall be reasonable to conclude that the fourth Gaussian-polynomial approximation is in most of cases more accurate than the Bagai or the saddlepoint approximations for approximating to the $1 \%$ significance level of the Bagai statistic. The method of polynomially adjusted Gaussian approximation provides at least comparable accuracy or outperforms the saddlepoint approximation for percentiles of interest. It should also be mentioned that for the cases of $n=9$ and 15, the Bagai approximation is better than other approximations.

\subsection{Approximating the $5 \%$ significance level}

Table 3 shows the numerical results for the saddlepoint and the tenth degree Gaussian-polynomial approximations in the case of a $5 \%$ significance level in addition to the exact probability and Bagai approximation calculated in Bagai et al. (1989). The differences between the exact probability of the Bagai statistic and the approximations are given in Table 4. The numerical details of the Gaussian- 
Table 4: Difference between the exact values and approximations

\begin{tabular}{|c|c|c|c|c|c|c|c|}
\hline$n$ & Bagai & Saddlepoint & GP(2) & GP(4) & GP(6) & GP(8) & GP(10) \\
\hline 5 & 0.0103 & 0.004630 & 0.007261 & 0.005646 & 0.006672 & 0.006119 & 0.005057 \\
\hline 6 & 0.0126 & 0.011221 & 0.012547 & 0.011726 & 0.012515 & 0.012015 & 0.011360 \\
\hline 7 & 0.0084 & 0.007625 & 0.008303 & 0.007901 & 0.008511 & 0.008091 & 0.007699 \\
\hline 8 & 0.0009 & 0.000031 & 0.000803 & 0.000191 & 0.000622 & 0.000268 & 0.000025 \\
\hline 9 & 0.0047 & 0.004254 & 0.004679 & 0.004388 & 0.004750 & 0.004455 & 0.004259 \\
\hline 10 & 0.0036 & 0.003158 & 0.003591 & 0.003266 & 0.003552 & 0.003300 & 0.003152 \\
\hline 11 & 0.0014 & 0.000798 & 0.001411 & 0.000903 & 0.001120 & 0.000903 & 0.000780 \\
\hline 12 & 0.0033 & 0.003039 & 0.003348 & 0.003105 & 0.003304 & 0.003118 & 0.003030 \\
\hline 13 & 0.0012 & 0.000657 & 0.001172 & 0.000729 & 0.000882 & 0.000719 & 0.000642 \\
\hline 14 & 0.0017 & 0.001347 & 0.001720 & 0.001401 & 0.001538 & 0.001395 & 0.001335 \\
\hline 15 & 0.0021 & 0.001769 & 0.002117 & 0.001815 & 0.001934 & 0.001807 & 0.001759 \\
\hline 16 & 0.0011 & 0.000650 & 0.001039 & 0.000694 & 0.000794 & 0.000681 & 0.000639 \\
\hline 17 & 0.0019 & 0.001452 & 0.001775 & 0.001488 & 0.001579 & 0.001478 & 0.001444 \\
\hline 18 & 0.0010 & 0.000722 & 0.001039 & 0.000754 & 0.000835 & 0.000743 & 0.000714 \\
\hline 19 & 0.0011 & 0.000852 & 0.001146 & 0.000888 & 0.000952 & 0.000870 & 0.000845 \\
\hline 20 & 0.0012 & 0.000922 & 0.001184 & 0.000946 & 0.001012 & 0.000937 & 0.000915 \\
\hline
\end{tabular}

polynomial approximants with the successive degrees used up to the optimal degree are also included in Table 4. The bolded numbers represent the minimum difference between the exact values and the approximations. We can see that the tenth degree Gaussian-polynomial approximant provides the minimum differences in 12 cases out of 16 cases, whereas the saddlepoint approximation is more accurate in only 4 cases. Tables 3 and 4 clearly indicate that the tenth degree Gaussian-polynomial approximation is in most of the cases more accurate than the Bagai and saddlepoint approximations in connection with approximating the $5 \%$ significance level of the Bagai statistic.

\subsection{Computational Issues}

The Gaussian-polynomial approximation is computationally simple and easy to program since it has very simple explicit expression unlike saddlepoint approximation. It should be pointed out that the Gaussian-polynomial approximation uses less information than the saddlepoint method, since Gaussian-polynomial approximation utilizes the same number of the first moments with the degree of polynomial adjustment, whereas saddlppoint approximation require the cumulant generating function from which all the moments can be generated. The selection for the optimal degree of the polynomial adjustment for obtaining a suitable approximation can be determined by making use of a de visu rule proposed in Ha and Provost (2007) among other critera. The tolerance was taken to be the precision of the saddlepoint approximation. In connection with the numerical precision for the Gaussian-polynomial distribution approximants given in Equation (3.10), the integration started from $-1,300$, which should give enough precision in terms of $10^{-6}$ decimals for all cases of interest. Figure 1 shows Bagai (dashed) and the $10^{\text {th }}$ degree Gaussian-polynomial (solid) approximations when $n=8$. Figure 2 shows that the difference between the Bagai approximation and the Gaussian-polynomial approximation with the $4^{\text {th }}$ (dashed) and $10^{\text {th }}$ (solid) degrees. The fluctuating behavior suggests that the proposed approximant evolves to adapt to the feature of the target density function. The symbolic computational package Mathematica was utilized for obtaining the numerical results.

\section{Acknowledgements}

The author wishes to express his sincere gratitude to the journal editor and two referees. The suggestions and comments of the referees pointed out crucial arguments on the initial version of manuscript, 


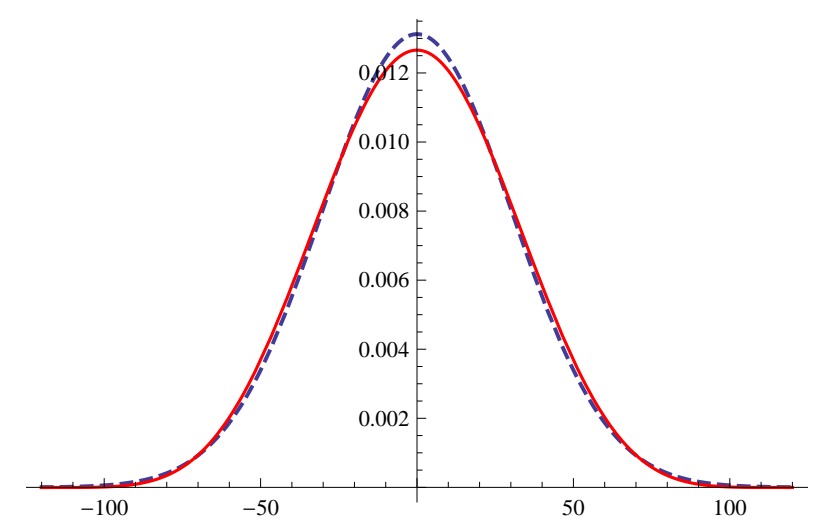

Figure 1: Bagai (dashed) and $10^{\text {th }}$ degree Gaussian-polynomial (solid) Approximations when $n=8$

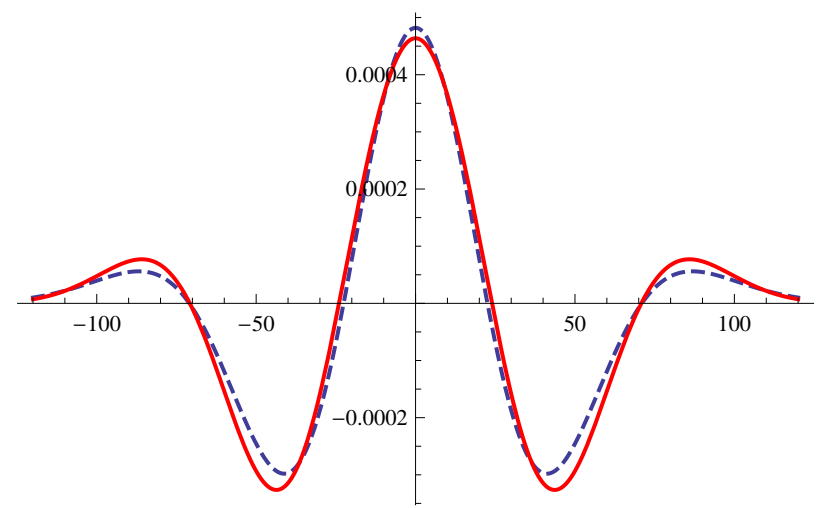

Figure 2: Differences Between Bagai and the $4^{\text {th }}$ (dashed) and $10^{\text {th }}$ (solid) degree Gaussian-Polynomial Approximations when $n=8$

which led to significant improvements on the quality of this paper.

\section{References}

Bagai, I., Deshpande, J. V. and Kochar, S. C. (1989). Distribution free tests for stochastic ordering in the competing risks model, Biometrika, 76, 775-781.

Bean, R., Froda, S. and Van Eeden, C. (2004). The normal, edgeworth, saddlepoint, uniform approximations to the Wilcoxon-Mann-Whitney null-distribution: A numerical comparison, Journal of Nonparametric Statistics, 16, 279-288.

Butler, R. W. (2007). Saddlepoint Approximations with Applications, Cambridge University Press.

Daniels, H. E. (1954). Saddlepoint approximations in statistics, Annals of Mathematical Statistics, 25, 631-650.

Daniels, H. E. (1987). Tail probability approximations, International Statistical Review, 55, 37-48.

Easton, G. S. and Ronchetti, E. (1986). General saddlepoint approximations with applications to L statistics, Journal of the American Statistical Association, 81, 420-430.

Goutis, C. and Casella, G. (1999). Explaining the saddlepoint approximation, American Statistician, 53, 216-224. 
Ha, H-T. (2007). Moment-based density approximation algorithm for symmetric distributions, The Korean Communications in Statistics, 14, 583-592.

Ha, H-T. and Provost, S. B. (2007). A viable alternative to resorting to statistical tables, Communication in Statistics: Simulation and Computation, 36, 1135-1151.

Huzurbazar, S. (1999). Practical saddlepoint approximations, American Statistician, 53, 225-232.

Jensen, J. L. (1995). Saddlepoint Approximations, Oxford University Press.

Kolassa, J. E. (2006). Series Approximation Methods in Statistics, Springer-Verlag.

Lugannani, R. and Rice, S. O. (1980). Saddlepoint approximation for the distribution of the sum of independent random variables, Advances in Applied Probability, 12, 475-490.

Murakami, H. (2009). A saddlepoint approximation to a distribution-free test for stochastic ordering in the competing risks model, REVSTAT - Statistical Journal, 7, 189-201.

Provost, S. B., Min, J. and Ha, H-T. (2009). Moment-based approximations of probability mass functions with applications involving order statistics, Communication in Statistics: Theory and Methods, 38, 1969-1981

Reid, N. (1988). Saddlepoint methods and statistical inference (with discussion), Statistical Science, 3, 213-238.

Wood, A. T. A., Booth, J. G. and Butler, R. W. (1993). Saddlepoint approximations to the CDF of some statistics with nonnormal limit distributions, Journal of the American Statistical Association, 88, 680-686.

Received December 12, 2011; Revised January 9, 2012; Accepted February 23, 2012 